\title{
Robust topological Hall effect driven by tunable noncoplanar magnetic state in Mn-Pt-In inverse tetragonal Heusler alloys
}

\author{
Bimalesh Giri, ${ }^{1}$ Arif Iqbal Mallick, ${ }^{2}$ Charanpreet Singh, ${ }^{1}$ P. V. Prakash \\ Madduri, ${ }^{1}$ Françoise Damay, ${ }^{3}$ Aftab Alam, ${ }^{2}$ and Ajaya K. Nayak ${ }^{1, *}$ \\ ${ }^{1}$ School of Physical Sciences, National Institute of Science Education and Research, HBNI, Jatni-752050, India \\ ${ }^{2}$ Department of Physics, Indian Institute of Technology Bombay, Mumbai- 400076, India \\ ${ }^{3}$ Laboratoire Léon Brillouin, CEA-CNRS, CEA Saclay, 91191 Gif-sur-Yvette, France
}

(Dated: September 1, 2020)

\begin{abstract}
Manipulation of magnetic ground states by effective control of competing magnetic interactions has led to the finding of many exotic magnetic states. In this direction, the tetragonal Heusler compounds consisting of multiple magnetic sublattices and crystal symmetry favoring chiral Dzyaloshinskii-Moriya interaction (DMI) provide an ideal base to realize non-trivial magnetic structures. Here, we present the observation of a large robust topological Hall effect (THE) in the multi-sublattice $\mathrm{Mn}_{2-x} \mathrm{PtIn}$ Heusler magnets. The topological Hall resistivity, which originates from the non-vanishing real space Berry curvature in the presence of non-zero scalar spin chirality, systematically decreases with decreasing the magnitude of the canting angle of the magnetic moments at different sublattices. With help of first principle calculations, magnetic and neutron diffraction measurements, we establish that the presence of a tunable non-coplanar magnetic structure arising from the competing Heisenberg exchanges and chiral DMI from the $\mathrm{D}_{2 d}$ symmetry structure is responsible for the observed THE. The robustness of the THE with respect to the degree of non-collinearity adds up a new degree of freedom for designing THE based spintronic devices.
\end{abstract}

\section{INTRODUCTION}

In recent times, the art of easy manipulation of noncollinear magnetic structures over the collinear ones has led to a drastic shift of focus on research involving next generation spintronic devices. For instance, the topologically stable non-collinear magnetic objects, e.g. skyrmions, can be engineered effectively at significantly lower current densities, thereby, providing an efficient way to manipulate the information stored in these logic/spintronic devices [1 4. In most of the cases, a basic requirement for the stabilization of these non-collinear magnetic states is the presence of chiral magnetic interaction, the Dzyaloshinskii-Moriya interaction (DMI), that develops from the broken inversion symmetry in certain class of chiral magnets [5, 9] and layered thin films [1012. Owing to the presence of non-trivial topological configurations, large topological Hall effect (THE) has been reported in these systems [12 17. The basis of the THE can be associated with a non-vanishing scalar spin chirality $(\mathrm{SSC}) \chi_{i j k}=\mathbf{S}_{\mathbf{i}} \cdot\left(\mathbf{S}_{\mathbf{j}} \times \mathbf{S}_{\mathbf{k}}\right)$, that corresponds to the solid angle $\Omega$ subtended by three spins $\mathbf{S}_{i}, \mathbf{S}_{j}$ and $\mathbf{S}_{k}$ on a unit sphere. Although measurement of THE has been extensively used to characterize topological magnetic objects, the manipulation of THE for its direct use in spintronics is never demonstrated.

For the realization of THE, the system must exhibits a non-vanishing scalar spin chirality, which can appear in magnetic materials with non-collinear and non-coplanar spin structures [18 20]. However, the lack of chiral magnetic symmetry can force a net vanishing THE in most of these systems. Rare examples of nonzero SSC has been reported in systems with special lattice structures such as pyrochlore, triangular lattices etc., with distinctive type of spin configurations [18, 21, 22]. Recent observation of THE in a perpendicularly magnetized system with interfacial DMI corroborates the importance of chiral magnetic interaction to achieve a non-zero THE [23]. Along this direction, only limited experimental findings are reported in recent literature. Our particular interest is $\mathrm{Mn}$ based tetragonal Heusler materials well known for their potential use in the field of spintronics $[9,24, \sqrt[26]{ }$. In this family of materials, a flexible tuning of the magnetic properties, such as, magnetization, magnetic anisotropy and Curie temperature $\left(T_{C}\right)$ can be realized by tuning the sublattice magnetic moments. In addition, the noncentrosymmetric tetragonal $\mathrm{Mn}_{2} Y Z$ compounds (where $Y=\mathrm{Pt}, \mathrm{Rh}, \mathrm{Ir}$ and $\mathrm{Ni}$ ) crystallizing in the space group I $\overline{4} \mathrm{~m} 2$ are potential candidates to host DMI that can give chiral magnetic interaction in the system $9,26,28$. To realize our goal we select the inverse tetragonal Heusler compound $\mathrm{Mn}_{2}$ PtIn as a starting compound that consists of two magnetic sublattices of Mn atoms. Here, we show that a tunable topological Hall effect can be achieved depending upon the degree of non-collinearity of the magnetic moments in the system.

\section{RESULTS AND DISCUSSION}

Calculations for different structural and magnetic configurations of the proposed $\mathrm{Mn}_{2-x}$ PtIn systems were carried out to find out the energetically most favourable crystal and magnetic configurations. In case of the parent $\mathrm{Mn}_{2} \mathrm{PtIn}$, a minimum energy state was found for the tetragonal space group I $\overline{4} \mathrm{~m} 2$. We have per- 

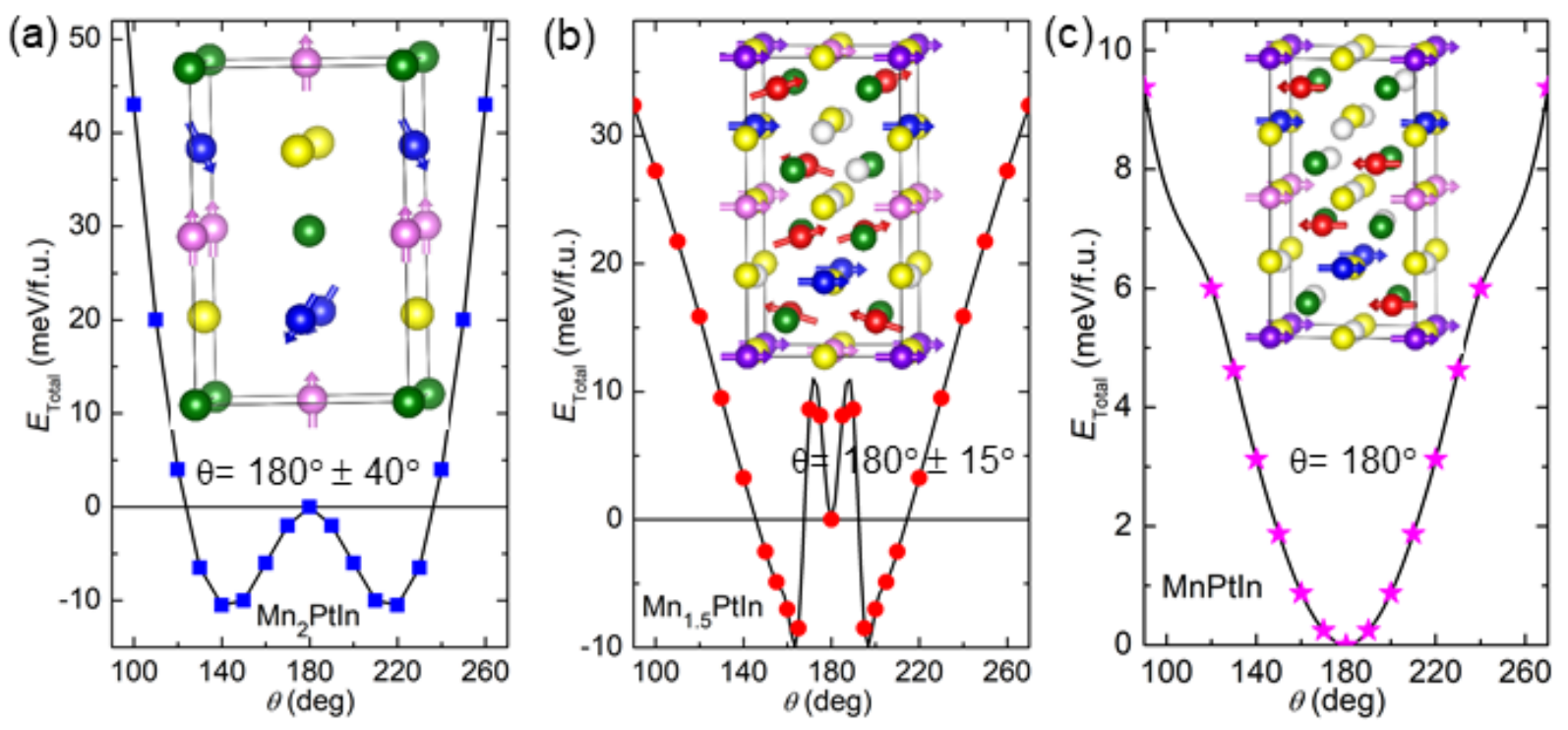

FIG. 1: (Color online) First principle calculation of magnetic structure for $\mathrm{Mn}_{2-x} \mathrm{PtIn}$. Total energy (with reference to $\mathrm{E}_{\theta=180^{\circ}}$ ) versus canting angle $\theta$ of the Mn moment in (a) $\mathrm{Mn}_{2} \mathrm{PtIn}$, (b) $\mathrm{Mn}_{1.5} \mathrm{PtIn}$ and (c) MnPtIn. The alignment of magnetic moment of each $\mathrm{Mn}$ atom in different magnetic sublattices are shown in the inset of respective figures. In case of $\mathrm{Mn}_{2} \mathrm{PtIn}$

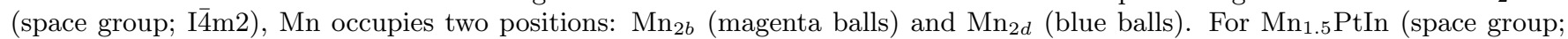
$\mathrm{I} \overline{4} 2 \mathrm{~m}$ ) and MnPtIn (space group; I $\overline{4} 2 \mathrm{~m}$ ) the $\mathrm{Mn}$ sits at $\mathrm{Mn}_{2 a}$ (violet balls), $\mathrm{Mn}_{2 b}$ (magenta balls), $\mathrm{Mn}_{4 d}$ (blue balls), Mn $\mathrm{b}_{8 i}$ (red balls). For all cases In and Pt atoms are represented by green and yellow balls, respectively.

formed fixed-direction magnetic calculations using the non-collinear module of the Vienna Ab-initio simulation package (VASP). From the total energy as a function of canting angle of Mn moments between the alternate layers [Fig. 1(a)], it is found that $\mathrm{Mn}_{2}$ PtIn exhibits a noncollinear magnetic state characterized by canting angle of $180^{\circ} \pm 40^{\circ}$. A maximum energy scale of about 0.35 $\mathrm{eV} /$ formula unit (f.u.) is the energy difference between the two collinear configurations - the ferromagnetic (FM) $\left(\theta=0^{\circ}\right)$ and the ferrimagnetic $(\mathrm{FiM})\left(\theta=180^{\circ}\right)$. The most significant exchange coupling is between the Mn atoms in the neighboring planes, i.e. between the $\mathrm{Mn}$ at $2 \mathrm{~b}$ and $2 \mathrm{~d}$ positions. The nearest neighbor exchange coupling aligns the Mn moments of the neighboring planes ferrimagnetically. The next nearest neighbor coupling between the $\mathrm{Mn}$ atoms sitting in $2 \mathrm{~d}$ position ( $\mathrm{Mn}-\mathrm{Pt}$ planes) is also significantly large and it also tries to align the Mn moments antiferromagnetically. The competition between these two interactions results in an effective canting of the Mn moments at the $2 \mathrm{~d}$ position. In case of $\mathrm{Mn}_{1.5}$ PtIn the most stable structural configuration was obtained by utilizing the experimental lattice parameters with the space group $\mathrm{I} \overline{4} 2 \mathrm{~m}$. $\mathrm{Mn}_{1.5} \mathrm{PtIn}$ exhibits a non-collinear magnetic order characterized by canting angle of $180^{\circ} \pm 15^{\circ}$ [Fig. 1(b)] and a total uncompensated moment of about $1.13 \mu_{B} /$ f.u. In case of MnPtIn, calculations were performed both for I $\overline{4} \mathrm{~m} 2$ and $\mathrm{I} \overline{4} 2 \mathrm{~m}$ space groups. In both the cases, there can be several possible structural configurations depending on the
Mn site occupancy. Irrespective of whatever magnetic configuration we start the calculation, it always stabilizes to a collinear magnetic arrangement for both the space groups [Fig. 1(c)]. A net cancellation of individual moments is achieved in case of for $\mathrm{I} \overline{4} \mathrm{~m} 2$, whereas a small uncompensated magnetic moment of $0.21 \mu_{B} /$ f.u. is found for $I \overline{4} 2 \mathrm{~m}$ space group.

Our experimental studies show that the parent $\mathrm{Mn}_{2} \mathrm{PtIn}$ crystallizes in an inverse Heusler tetragonal phase with space group İ̄m2 29. $\mathrm{Mn}_{2-x}$ PtIn with $x=$ 0.2 to 0.4 exhibit a mixed structural phases due to composition dependent structural transition from the space group $\mathrm{I} \overline{4} \mathrm{~m} 2$ to $\mathrm{I} \overline{4} 2 \mathrm{~m}$. Samples with $x=0.5$ to 1.0 crystallize in the space group $\bar{I} \overline{4} 2 \mathrm{~m}$ with a small fraction of MnPt phase for $x=0.9$ and 1.0 [29]. The isothermal magnetization $M(H)$ loops measured at $2 \mathrm{~K}$ for different $\mathrm{Mn}_{2-x}$ PtIn samples are plotted in Fig. 2(a). The saturation magnetization intially increases for $x=0$ to 0.3 before decreasing systematically with decreasing $\mathrm{Mn}$ concentration. As suggested by our theoretical calculations, a fully compensated magnetic state can be found for MnPtIn that exhibit a linear kind of hysteresis loop. The variation of saturation magnetization with the Mn concentration for $x=0.0$ to 1.0 is plotted in the inset of Fig. 2(a). The temperature dependence of the magnetization for the $\mathrm{Mn}_{2-x}$ PtIn are shown in the Fig. 2(b). As it can be seen, the Curie temperature $\left(T_{C}\right)$ systematically decreases with decreasing Mn concentration.

Our theoretical calculations and experimental studies 


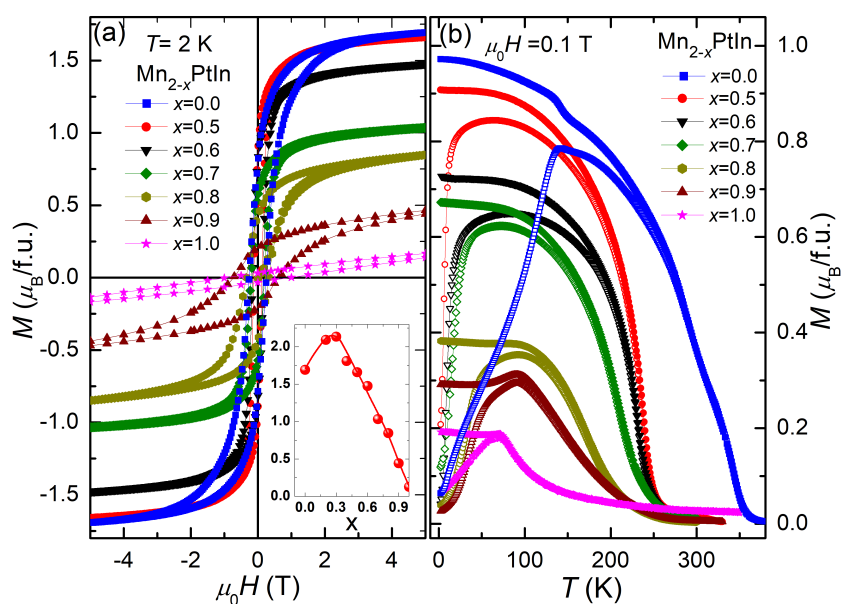

FIG. 2: (Color online) (a) Field dependence of magnetization loops measured at $2 \mathrm{~K}$ for $\mathrm{Mn}_{2-x} \mathrm{PtIn}$. The inset shows compositional dependent magnetization at a field of $5 \mathrm{~T}$. (b) Temperature dependence of magnetization $M(T)$ measured in zero field cooled (ZFC, open symbols) and field cooled (FC, closed symbols) modes in an applied field of $0.1 \mathrm{~T}$ for $\mathrm{Mn}_{2-x} \mathrm{PtIn}$. The $M(T)$ data for $\mathrm{x}=0.9$ and 1.0 are multiplied by a factor of 3 and 30, respectively, for a clear view.

suggest the presence of a tunable non-collinear magnetic state in the present system. To explore the effect of this non-collinearity, we have carried out a detailed Hall resistivity $\left(\rho_{y x}\right)$ measurements for $\mathrm{Mn}_{2} \mathrm{PtIn}$ as plotted in Fig. 3(a)-(f). It is noteworthy to mention here that an asymmetric behavior of $\rho_{y x}$ at $T=5 \mathrm{~K}$ mainly arises due to small longitudinal magnetoresistance contribution at this temperature, whereas, no such effect is found for $T \geq 50 \mathrm{~K}$. The total Hall resistivity in a system can be written as $\rho_{y x}=\rho_{N}+\rho_{A H}+\rho_{y x}^{T}$, where $\rho_{N}, \rho_{A H}$ and $\rho_{y x}^{T}$ are normal, anomalous and topological Hall resistivities, respectively. The normal Hall resistivity which is linearly proportional to the magnetic field can be expressed as $\rho_{N}=R_{0} H$, where $R_{0}$ is the normal Hall coefficient and $H$ is the magnetic field. In a FM/FiM system, the intrinsic contribution to the anomalous Hall effect can be illustrated as $\rho_{A H}=b \rho_{x x}^{2} M$, where $b$ is a constant, $\rho_{x x}$ is the longitudinal resistivity and $M$ is the magnetization. As can be seen from the experimental Hall resistivity data, at higher fields $\rho_{y x}$ almost saturates with fields. So, it can be assumed that the high field data only consists of the normal and anomalous Hall components. Therefore, $\rho_{y x}$ at high fields can be written as $\rho_{y x}=R_{0} H+b \rho_{x x}^{2} M$. The calculated Hall resistivity curves for different samples and temperatures sans topological Hall resistivity are plotted as solid lines in Fig. 3(a)-(h). In case of $\mathrm{Mn}_{2} \mathrm{PtIn}$, a significant difference between the experimental and calculated Hall resistivity data can be found for temperatures up to $100 \mathrm{~K}$ [Figs. 3(a)-(c)], where the $\rho_{y x}$ data exhibit a negative hysteresis loop. This suggests that for temperature range of $5 \mathrm{~K}$ to $100 \mathrm{~K}$, the addi-
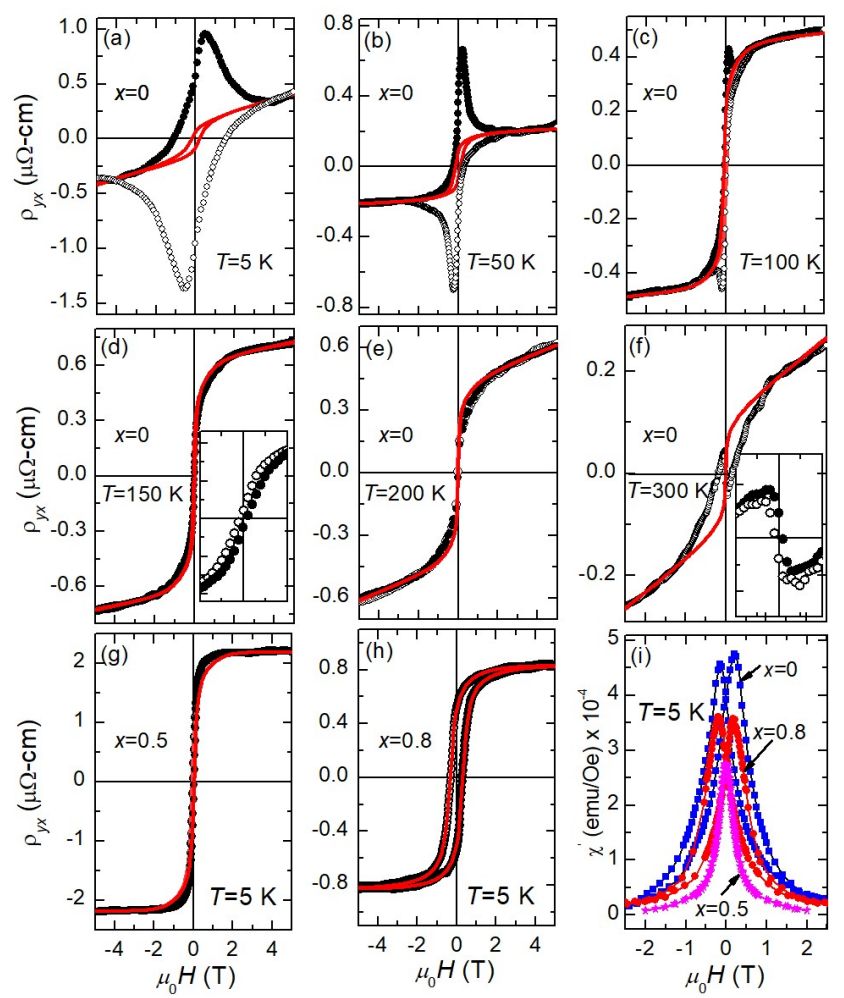

FIG. 3: (Color online) Field dependence of Hall resistivity $\left(\rho_{y x}\right)$ measured at different temperatures for (a)-(f) $\mathrm{Mn}_{2} \mathrm{PtIn},(\mathrm{g}) \mathrm{Mn}_{1.5} \mathrm{PtIn}$ and (h) $\mathrm{Mn}_{1.2} \mathrm{PtIn}$. The open and closed symbols represent experimental data with field sweep in $+H \rightarrow-H$ and $-H \rightarrow+H$, respectively. The solid lines corresponds to the total calculated Hall resistivity as described in the main text. (i) Field dependence of the real component of ac-susceptibility, $\chi^{\prime}(H)$ measured at $5 \mathrm{~K}$.

tional scattering of the conduction electrons takes place opposite to that of normal and anomalous Hall contribution. For $T \geq 150 \mathrm{~K}$, the experimental $\rho_{y x}$ data exhibit positive hysteresis loop as shown in the inset of Fig. 3(d) and 3(f). We also observe a reasonable difference between the experimental and calculated Hall resistivity data for $\mathrm{Mn}_{1.5}$ PtIn as shown in Fig. 3(g), whereas, both curves matches well for $\mathrm{Mn}_{1.2} \mathrm{PtIn}$, signifying the absence of any additional component of $\rho_{y x}$ for this sample [Fig. 3(h)].

To further understand the source of the observed anomaly in the Hall data, we have performed field dependent AC-susceptibility measurements at $5 \mathrm{~K}$ for all the three samples [Fig. 3(i)]. The $\chi^{\prime}(H)$ curves do not exhibit any kind of anomaly up to a field of $\pm 5 \mathrm{~T}$. It can be mentioned here that all skyrmion hosting bulk materials display dip/kink kind of features in the acsusceptibility measurements $7,26,30,32$. In addition, the first derivative of magnetization with respect to field for the $M(H)$ loops also do not exhibit any unusual behavior [29]. Therefore, it is very unlikely that the present samples possess any kind of skyrmionic phase. 


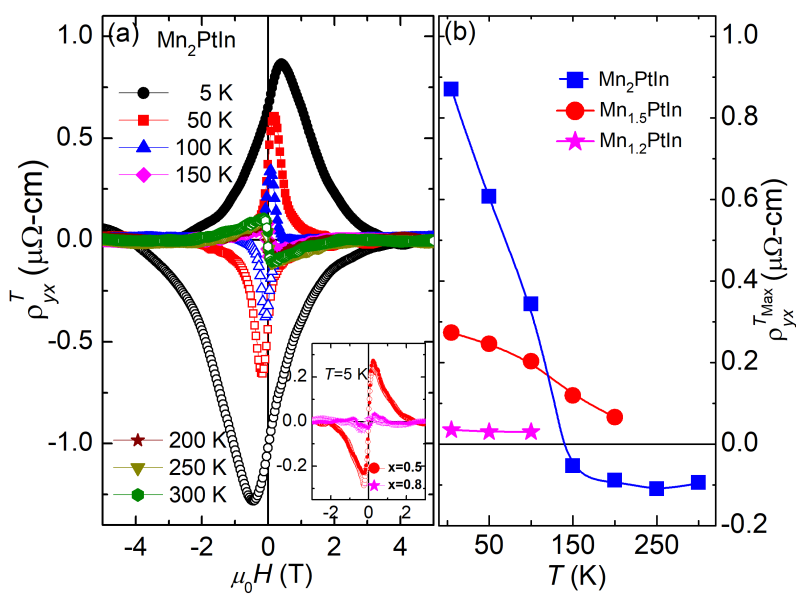

FIG. 4: (Color online) (a) Topological Hall resistivity $\left(\rho_{y x}^{T}\right)$ calculated at different temperatures for $\mathrm{Mn}_{2} \mathrm{PtIn}$. The open and closed symbols represent experimental data with field sweep in $+H \rightarrow-H$ and $-H \rightarrow+H$, respectively. The inset shows calculated $\rho_{y x}^{T}$ at $5 \mathrm{~K}$ for $\mathrm{Mn}_{1.5}$ PtIn (filled circles) and $\mathrm{Mn}_{1.2}$ PtIn (filled stars) in $\mu \Omega-\mathrm{cm}$. (b) Maximum value of $\rho_{y x}^{T}$ as a function of temperatures (solid symbols) taken from the field dependent $\rho_{y x}^{T}$ data.

The calculated $\rho_{y x}\left(=\rho_{N}+\rho_{A H}\right)$ was subtracted from the experimental total Hall resistivity to obtain the topological Hall $\rho_{y x}^{T}$. For $\mathrm{Mn}_{2} \mathrm{PtIn}$, it is found that for $T \leq 100 \mathrm{~K}$, the maximum value of $\rho_{y x}^{T}$ lies in the first and the third quadrants, whereas, the maxima lies in the second and fourth quadrants for $T \geq 150 \mathrm{~K}$ [Fig. 4(a)]. A large $\rho_{y x}^{T}$ of about $1 \mu \Omega-\mathrm{cm}$ can be found at $5 \mathrm{~K}$ in $\mathrm{Mn}_{2} \mathrm{PtIn}$. The $\rho_{y x}^{T}$ drastically decreases to about $0.3 \mu \Omega-\mathrm{cm}$ in case of $\mathrm{Mn}_{1.5}$ PtIn before vanishing for $\mathrm{Mn}_{1.2} \mathrm{PtIn}$, as depcted in inset of Fig. 4(a). Most importantly, $\rho_{y x}^{T}$ displays a strong correlation with the magnitude of canting angle in the system. To understand the chnage in sign of the $\rho_{y x}^{T}$ above $150 \mathrm{~K}$ for $\mathrm{Mn}_{2} \mathrm{PtIn}$, we have plotted maximum value of $\rho_{y x}^{T}$ taken from the field sweep $-H \rightarrow+H$ at different temperatures [Fig. 4(b)]. The origin of the change in sign of the $\rho_{y x}^{T}$ can be attributed to the existence of a spin-reorientation like transition at $150 \mathrm{~K}$ as can be visualized in ZFC and FC $M(T)$ curves shown earlier in Fig. 2(b). In case of $\mathrm{Mn}_{1.5} \mathrm{PtIn}$ the maximum value of $\rho_{y x}^{T}$ monotonically decreases with temperature, whereas, it remains almost close to zero at all temperatures for $\mathrm{Mn}_{1.2} \mathrm{PtIn}$. It is important to mention here that the $M(T)$ data for both $\mathrm{Mn}_{1.5} \mathrm{PtIn}$ and $\mathrm{Mn}_{1.2}$ PtIn do not exhibit any kind of anomaly [see Fig. $2(\mathrm{~b})]$.

Finally, to experimentally verify the existence of noncollinear magnetic structure in the present system we have performed powder neutron diffraction (ND) experiment on $\mathrm{Mn}_{1.5} \mathrm{PtIn}$. The ND patterns taken in the temperature range of $1.5 \mathrm{~K}$ to $300 \mathrm{~K}$ at different $2 \theta$ values are
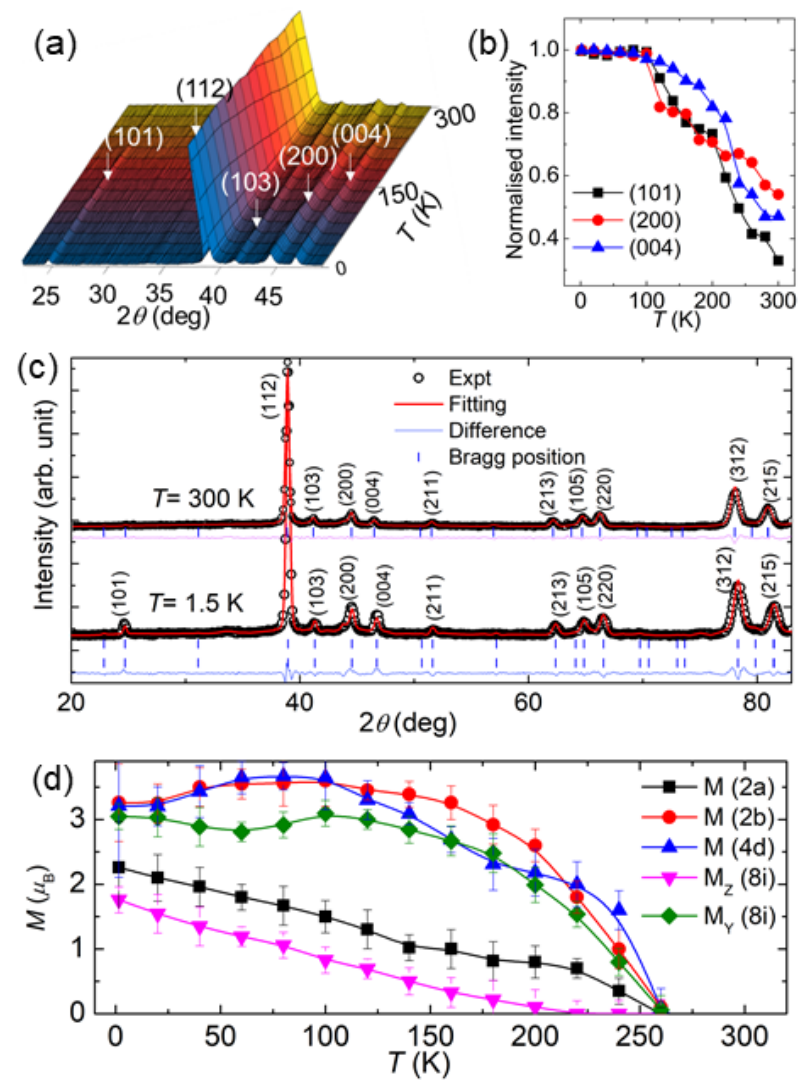

FIG. 5: (Color online) (a) Neutron diffraction patterns for $\mathrm{Mn}_{1.5}$ PtIn measured at different temperatures. For a clear view of the major magnetic reflections the patterns are shown in the $2 \theta$ range of 22 degree to 50 degree. (b) Temperature variation of normalized integrated intensity for the three major magnetic reflections (101), (200) and (004). (c) Rietveld refinement of the neutron diffraction patterns at $300 \mathrm{~K}$ and $1.5 \mathrm{~K}$ for $\mathrm{Mn}_{1.5}$ PtIn. (d) Temperature dependence of net magnetic moment of site-specific Mn atoms at different sublattices as depicted by different symbols.

depicted in Fig. 5(a). The temperature dependent ND patterns show an increase in the scattering intensity below the ordering temperature at the nuclear Bragg peaks (101), (200) and (004), suggesting a commensurate magnetic structure. This can be clearly seen from the temperature variation of normalized intensities which decrease significantly with increasing temperature, suggesting the presence of contribution from both in-plane and out-ofplane magnetic components [Fig. 5(b)]. Since $\mathrm{Mn}_{1.5} \mathrm{PtIn}$ exhibits a $T_{C}$ of about $240 \mathrm{~K}$, the ND pattern at $300 \mathrm{~K}$ used for the nuclear refinement by utilizing the previously determined space group I $\overline{4} 2 \mathrm{~m}$ (SG No. 121) and related structural parameters [Fig. 5(c), upper panel]. Furthermore, we obtained the magnetic propagation vector $k=(0,0,0)$ with best agreement factors by using the $k$-search programme included in Fullprof-suite package. The Rietveld refinement of $1.5 \mathrm{~K} \mathrm{ND}$ data convincingly demonstrates the presence of magnetic contribution in 

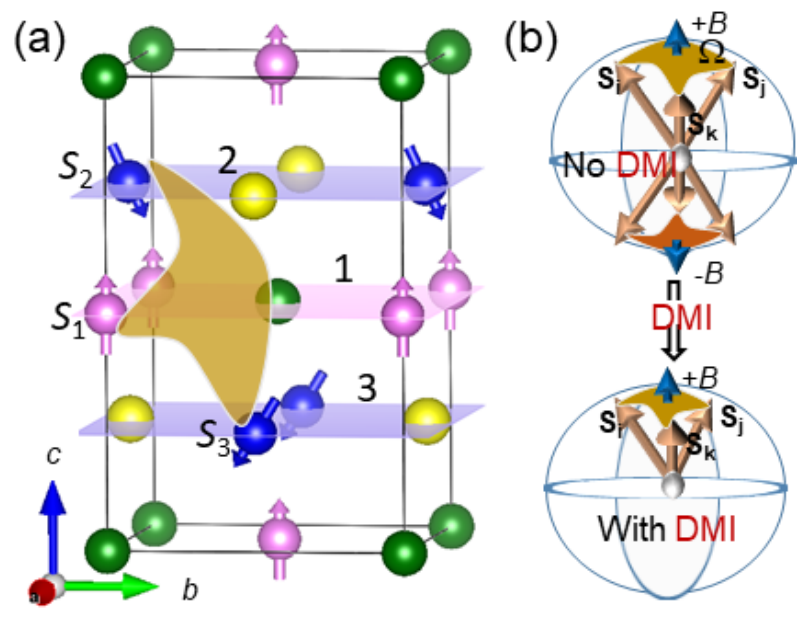

FIG. 6: (Color online) (a) Unit cell for $\mathrm{Mn}_{2}$ PtIn representing $\mathrm{Mn}$ moments at different lattice planes. Mn-In and $\mathrm{Mn}-\mathrm{Pt}$ lattice planes are shown in light magenta color marked by 1 and light blue color marked by 2 and 3, respectively. Solid angle subtaineded by three moments $\mathbf{S}_{\mathbf{1}}, \mathbf{S}_{\mathbf{2}}$ and $\mathbf{S}_{\mathbf{3}}$ is ahown in dark yellow color. (b) Upper panel; solid angle $\Omega$ subtended by three non-coplanar spins $\mathbf{S}_{\mathbf{i}}, \mathbf{S}_{\mathbf{j}}, \mathbf{S}_{\mathbf{k}}$ that gives a fictitious magnetic field in both upward and downward direction (blue arrows) in the absence of any chiral DMI. Lower panel; fixed chirality in the presence of DMI.

$\mathrm{Mn}_{1.5}$ PtIn [Fig. 5(c), lower panel]. The temperature dependence of absolute values of the magnetic moments for Mn sitting at different sublattices are shown in Fig. 5(d). The Mn sitting at 2b, 4d and 8i display almost equal magnitudes of magnetic moments with similar temperature dependance. The Mn moments at $2 \mathrm{a}, 2 \mathrm{~b}$ and $4 \mathrm{~d}$ exhibit a complete in-plane orientation, whereas, $8 \mathrm{i} \mathrm{Mn}$ atoms possess both in-plane and out-of-plane magnetic components. A smaller magnitude of $2 \mathrm{a} \mathrm{Mn}$ moment is due to the fact that 2a site is comparatively less occupied in the present sample. More details about the analysis of ND data can be found from the supplementary information [29.

Our theoretical calculation and experimental results have convincingly established the presence of noncoplanar magnetic state in the present system. For a better understanding of the present THE that originates from the non-vanishing scalar spin chirality, we have considered one $\mathrm{Mn}$ spin $\left(\mathbf{S}_{\mathbf{1}}\right)$ in the Mn-In plane (plane 1) and two Mn spins $\left(\mathbf{S}_{\mathbf{2}}\right.$ and $\mathbf{S}_{\mathbf{3}}$ ) from two different $\mathrm{Mn}-\mathrm{Pt}$ planes (plane 2 and 3 ) as shown in Fig. 6(a). For simplicity, first we consider the components of the canted spins $\mathbf{S}_{\mathbf{2}}$ and $\mathbf{S}_{\mathbf{3}}$ lie in $y-z$ plane. The magnetic moment of $\mathrm{Mn}$ atoms sitting in plane 1, 2 and 3 can be described by $\mathbf{S}_{\mathbf{1}}$ $=Z_{1} \mathbf{k}, \mathbf{S}_{\mathbf{2}}=Y_{2} \mathbf{j}-Z_{2} \mathbf{k}, \mathbf{S}_{\mathbf{3}}=-Y_{2} \mathbf{j}-Z_{2} \mathbf{k}$, respectively. Here $Y_{2}$ is the component of Mn moment in the $y$ direction and $Z_{1}$ and $Z_{2}$ are that of $z$ direction. $\mathbf{i}, \mathbf{j}, \mathbf{k}$ are the unit vectors. For the said configuration the scalar spin chirality can be calculated as $\chi_{123}=\mathbf{S}_{\mathbf{1}} \cdot\left(\mathbf{S}_{\mathbf{2}} \times \mathbf{S}_{\mathbf{3}}\right)=0$.
However, the competing antiferromagnetic interactions along with the chiral DMI in the system and/or the external magnetic field can tilt the in-plane component of the $\mathbf{S}_{\mathbf{2}}$ and $\mathbf{S}_{\mathbf{3}}$ in any direction in the $a b$ plane. Hence, with a small $x$ component $\delta, \mathbf{S}_{\mathbf{2}}=\delta \mathbf{i}+Y_{2} \mathbf{j}-Z_{2} \mathbf{k}$ and $\mathbf{S}_{\mathbf{3}}=\delta \mathbf{i}-Y_{2} \mathbf{j}-Z_{2} \mathbf{k}$. As a result, we can achieve a nonvanishing $\mathbf{S}_{\mathbf{1}} \cdot\left(\mathbf{S}_{\mathbf{2}} \times \mathbf{S}_{\mathbf{3}}\right)=-2 Y_{2} Z_{1} \delta$ that can give rise to the observed topological Hall effect. As schematically depicted in Fig. 6(b), any three non-coplanar spins $\mathbf{S}_{\mathbf{i}}$, $\mathbf{S}_{\mathbf{j}}$ and $\mathbf{S}_{\mathbf{k}}$ can subtend a solid angle $\Omega$, thereby resulting in a non-zero scalar spin chirality with a fictitious magnetic field as shown by blue arrow. In absence of any fixed chirality this magnetic field will act in all possible directions resulting in a net vanishing THE. However, the $D_{2 d}$ symmetry of the present materials ensures a chiral magnetic state, thereby, a non-vanishing THE in the system. At very high magnetic fields the Zeeman energy can easily overcome the chiral DMI energy, suppressing the chirality as well as the THE.

Our assertion of large topological Hall resistivity at low temperatures as a result of finite scalar spin chirality instead of any skyrmion phase is supported by several facts. (i) We do not observe any kind of kink/peak behavior in the ac-susceptibility measurements as discussed earlier. (ii) The isostructural Heusler compounds $\mathrm{Mn}_{1.4} \mathrm{Pt}_{0.9} \mathrm{Pd}_{0.1} \mathrm{Sn}$ [9] and $\mathrm{Mn}_{2} \mathrm{Rh}_{0.9} \mathrm{Ir}_{0.1} \mathrm{Sn}$ 33 found to exhibit antiskyrmion phase with antiskyrmion size of about $150 \mathrm{~nm}$ and $200 \mathrm{~nm}$, respectively . Since the magnitude of THE is inversely proportional to the skyrmion size (density), it is expected that the antiskyrmion phase in these systems will result in topological Hall resistivity in the order of $1 \mathrm{n} \Omega-\mathrm{cm}$ or less. (iii) It can be clearly seen that $\mathrm{Mn}_{2}$ PtIn displays a spin-reorientation transition around $150 \mathrm{~K}$. The previous studies on similar systems show the existence of antiskyrmion phase, if any, only above the spin-reorientation transition [9, 32.

The non-coplanar spin structure with finite scalar spin chirality as a source of THE has been recently observed in FM systems [23], as well as in antiferromagnetic systems (AFM) 34. Although THE arises in systems hosting skyrmions/antiskyrmions or comprised of non-coplanar spin structures, a very basic difference lies in the length scale of the periodicity associated with their magnetic structure. A crossover between a long periodic magnetic structure to a comparatively shorter scale non-coplanar magnetic state is about interplay between the energy contributions from various energy terms [35. In case of the present systems under study, it might be possible to stabilize a incommensurately modulated heli$\mathrm{cal} /$ cycloid ground state with a modulation period up to few hundreds. These helix/cycloid can transform into skyrmions/antiskyrmions under the external magnetic field and in turn can cause very small THE due to the large size of the skyrmions. In a recent study, Kumar et $a l$. have assigned the low temperature THE in some of their samples to the presence of antiskyrmions, although 
these samples display a large canting angle below the spin-reorientation transition [36]. However, the THE is only found when there is a large canting angle, indicating its probable origin from the non-vanishing scalar spin chirality coming from the non-coplanar magnetic state. This scenario is supported by non existence of any THE in the well-established antiskyrmion phase, probably due to the large size of the antiskyrmions in these materials.

\section{CONCLUSION}

In summary, our theoretical calculations as well as exprimental findings convincingly establish the presence of non-collinear magnetic ground states, resulting in a robust THE. We show a controlled tuning of the topological Hall effect by modifying the canted magnetic state in the system. The magnitude of the spin canting achieved by tuning the Mn composition is associated with a small change in lattice parameters that eventually controls various fundamental parameters such as the exchange interactions, the DMI, and the magnetocrystalline anisotropy (MCA). Hence, the competition among these parameters determines the underlying magnetic texture of the present system. Therefore, the THE in the present case can be controlled electrically by inducing strain in the system. Hence, the present study on realization of tunable THE possesses a great potential in all electrical switching based memory application. A very recent study on electrical control of anomalous Hall state corroborate the importance of the present study that can motivate further research in this direction [37].

AKN acknowledges the support from Department of Atomic Energy (DAE), the Department of Science and Technology (DST)-Ramanujan research grant (No. SB/S2/RJN-081/2016), SERB research grant (ECR/2017/000854) and Nanomission research grant [SR/NM/NS-1036/2017(G)] of the Government of India. AA thank IRCC, IIT Bombay for supporting this research through Early Carrier Award grant, Code: RI/0217/10001338-001.

* Electronic address: ajaya@niser.ac.in

[1] F. Jonietz, S. Mhlbauer, C. Pfleiderer, A. Neubauer, W. Mnzer, A. Bauer, T. Adams, R. Georgii, P. Bni, R. A. Duine, K. Everschor, M. Garst, and A. Rosch, Science 330, 1648 (2010).

[2] X. Z. Yu, N. Kanazawa, W. Z. Zhang, T. Nagai, T. Hara, K. Kimoto, Y. Matsui, Y. Onose, and Y. Tokura, Nat. Commun. 3, 988 (2012).

[3] N. Nagaosa and Y. Tokura, Nat. Nanotechnol. 8, 899 (2013).

[4] A. Fert, V. Cros, and J. Sampaio, Nat. Nanotechnol. 8, 152 (2013).
[5] S. Mühlbauer, B. Binz, F. Jonietz, C. Pfleiderer, A. Rosch, A. Neubauer, R. Georgii, and P. Böni, Science 323, 915 (2009).

[6] X. Z. Yu, Y. Onose, N. Kanazawa, J. H. Park, J. H. Han, Y. Matsui, N. Nagaosa, and Y. Tokura, Nature 465, 901 (2010).

[7] S. Seki, X. Z. Yu, S. Ishiwata, and Y. Tokura, Science 336, 198 (2012).

[8] C. Jin, Z.-A. Li, A. Kovacs, J. Caron, F. Zheng, F.N. Rybakov, N.S. Kiselev, H. Du, S. Blügel, M. Tian, Y. Zhang, M. Farle, and R. E. Dunin-Borkowski, Nat. Commun. 8, 15569 (2017).

[9] A. K. Nayak, V. Kumar, T. Ma, P. Werner, E. Pippel, R. Sahoo, F. Damay, U. K. Rößler, C. Felser, and S. S. P. Parkin, Nature 548, 561 (2017).

[10] S. Heinze, K. von Bergmann, M. Menzel, J. Brede, A. Kubetzka, R. Wiesendanger, G. Bihlmayer, and S. Bügel, Nat. Phys. 7, 713 (2011).

[11] A. Soumyanarayanan, M. Raju, A. L. G. Oyarce, A. K. C. Tan, M.-Y. Im, A. P. Petrovi, P. Ho, K. H. Khoo, M. Tran, C. K. Gan, F. Ernult, and C. Panagopoulos, Nat. Mater. 16, 898 (2017).

[12] M. Raju, A. Yagil, A. Soumyanarayanan, A. K. C. Tan, A. Almoalem, F. Ma, O. M. Auslaender, and C. Panagopoulos, Nat. Commun. 10, 696 (2019).

[13] S.R. Saha, H. Sugawara, T.D. Matsuda, H. Sato, R. Mallik, and E. V. Sampathkumaran, Phys. Rev. B 60, 12162 (1999).

[14] A. Neubauer, C. Pfleiderer, B. Binz, A. Rosch, R. Ritz, P. G. Niklowitz, and P. Bni, Phys. Rev. Lett. 102, 186602 (2009).

[15] N. Kanazawa, Y. Onose, T. Arima, D. Okuyama, K. Ohoyama, S. Wakimoto, K. Kakurai, S. Ishiwata, and Y. Tokura, Phys. Rev. Lett. 106, 156603 (2011).

[16] J. C. Gallagher, K. Y. Meng, J. T. Brangham, H. L. Wang, B. D. Esser, D. W. McComb, and F. Y. Yang, Phys. Rev. Lett. 118, 027201 (2017).

[17] T. Kurumaji, T. Nakajima, M. Hirschberger, A. Kikkawa, Y. Yamasaki, H. Sagayama, H. Nakao, Y. Taguchi, T. Arima, and Y. Tokura, Science 365, 914 (2019).

[18] Y. Taguchi, Y. Oohara, H. Yoshizawa, N. Nagaosa, and Y. Tokura, Science 291, 2573 (2001).

[19] H. Ishizuka and N. Nagaosa, Sci. Adv. 4, eaap9962 (2018).

[20] P. K. Rout, P. V. P. Madduri, S. K. Manna and A. K. Nayak, Phys. Rev. B 99, 094430 (2019).

[21] Y. Machida, S. Nakatsuji, Y. Maeno, T. Tayama, T. Sakakibara, and S. Onoda, Phys. Rev. Lett. 98, 057203 (2007).

[22] H. Takatsu, S. Yonezawa, S. Fujimoto, and Y. Maeno, Phys. Rev. Lett. 105, 137201 (2010).

[23] W. Wang, M. W. Daniels, Z. Liao, Y. Zhao, J. Wang, G. Koster, G. Rijnders, C.-Z. Chang, D. Xiao, and W. Wu, Nat. Mater. 18, 1054 (2019).

[24] T. Graf, C. Felser, and S. S. Parkin, Progress in Solid State Chemistry 39, 1 (2011).

[25] A. K. Nayak, M. Nicklas, S. Chadov, P. Khuntia, C. Shekhar, A. Kalache, M. Baenitz, Y. Skourski, V. K. Guduru, A. Puri, U. Zeitler, J. M. D. Coey, and C. Felser, Nat. Mater. 14, 679 (2015).

[26] S. Sen, C. Singh, P. K. Mukharjee, R. Nath, and A. K. Nayak, Phys. Rev. B 99, 134404 (2019).

[27] A. K. Nayak, C. Shekhar, J. Winterlik, A. Gupta, and 
C. Felser, Appl. Phys. Lett. 100, 152404 (2012).

[28] O. Meshcheriakova, S. Chadov, A. K. Nayak, U. K. Rößler, J. Kübler, G. André, A. A. Tsirlin, J. Kiss, S. Hausdorf, A. Kalache, W. Schnelle, M. Nicklas, and C. Felser, Phys. Rev. Lett. 113, 087203 (2014).

[29] See Supplemental Material at http://link.aps.org/supplemental/ 10.1103/PhysRevB.102.014449 for details of sample preparation, characterization, magnetic and transport measurements, and neutron diffraction analysis, which includes Refs. $[15,3846]$.

[30] H. Wilhelm, M. Baenitz, M. Schmidt, U. K. Rössler, A. A. Leonov, and A. N. Bogdanov, Phys. Rev. Lett. 107, 127203 (2011).

[31] A. Bauer and C. Pfleiderer, Phys. Rev. B 85, 214418 (2012)

[32] Sk Jamaluddin, S. K. Manna, B. Giri, P. V. Prakash Madduri, S. S. P. Parkin, and A. K. Nayak, Adv. Funct. Mater. 29, 1901776 (2019).

[33] J. Jena, R. Stinshoff, R. Saha, A. K. Srivastava, T. Ma, H. Deniz, P. Werner, C. Felser, and S. S. P. Parkin, Nano Lett. 20, 59 (2020).

[34] C. Sürgers, G. Fischer, P. Winkel, and H. von Löhneysen, Nat. Commun. 5, 3400 (2014).

[35] A. N. Bogdanov, U. K. Rößler, M. Wolf, and K.-H. Müller, Phys. Rev. B 66, 214410 (2002).

[36] V. Kumar, N. Kumar, M. Reehuis, J. Gayles, A. S. Sukhanov, A. Hoser, F. Damay, C. Shekhar, P. Adler, and C. Felser, Phys. Rev. B 101, 014424 (2020).

[37] H. Tsai, T. Higo, K. Kondou, T. Nomoto, A. Sakai, A. Kobayashi, T. Nakano, K. Yakushiji, R. Arita, S. Miwa, Y. Otani, and S. Nakatsuji, Nature 580, 608 (2020).

[38] G. Kresse and J. Furthmuller, Phys. Rev. B 54, 11169 (1996); Comput. Mater. Sci. 6, 15 (1996).

[39] G. Kresse and D. Joubert, Phys. Rev. B 59, 1758 (1999).

[40] L. Pál, E. Krén, G. Kádár, P. Szabó, and T. Tarnóczi, J. App. Phys. 39, 538 (1968).

[41] A.S. Wills, Physica B 276, 680 (2000).

[42] N. Nagaosa, J. Sinova, S. Onoda, A. H. MacDonald, and N. P. Ong, Rev. Mod. Phys. 82, 1539 (2010).

[43] Y. Tian, L. Ye, and X. Jin, Phys. Rev. Lett. 103, 087206 (2009).

[44] S. X. Huang and C. L. Chien, Phys. Rev. Lett. 108, 267201 (2012).

[45] K. Ueda, R. Kaneko, H. Ishizuka, J. Fujioka, N. Nagaosa, and Y. Tokura, Nat. Commun. 9, 3032 (2018).

[46] M. Lee, Y. Onose, Y. Tokura, and N.P. Ong, Phys. Rev. B 75, 172403 (2007).

\section{SUPPLEMENTARY INFORMATION}

\section{METHODS}

Polycrystalline ingots of $\mathrm{Mn}_{2-x} \mathrm{PtIn}$ with $\mathrm{x}=0.0$ to1.0 were prepared by arc melting technique using the stoichiometric amounts of high purity constituent elements in a high purity argon atmosphere. To ensure a better homogeneity, the samples were melted multiple times by flipping upside down. The ingots were sealed in an evacuated quartz tube and subsequently annealed at $1023 \mathrm{~K}$ for seven days followed by quenching in ice-water mixture. The structural phase of the annealed samples was determined by room temperature powder X-ray diffraction (XRD) using $\mathrm{Cu}-\mathrm{K}_{\alpha}$ radiation (Rigaku). The homogeneity and composition of the samples were determined by field emission scanning electron microscopy (FESEM), energy-dispersive X-ray spectroscopy (EDS). Magnetic measurements were carried out using vibrating sample SQUID magnetometer (MPMS-3, Quantum Design). Transport measurements were carried out using Quantum Design Physical Property Measurement System (PPMS). Powder neutron diffraction measurements were performed in Laboratoire Léon Brillouin-Orphee, CEA-CNRS Saclay, using G4.1 set up with neutron wavelength of $2.426 \AA$. Theoretical calculations were performed using density functional theory (DFT) as implemented within the Vienna ab-initio simulation package (VASP) 38, with a projected augmented basis 39. The exchange-correlation functionals used for the noncollinear magnetic calculations are based on the local density approximation (LDA). A reasonably high planewave cut-off of $400 \mathrm{eV}$ and a Monkhorst-Pack k -point mesh with reasonable density for different unit cells were used for the calculations.

\section{STRUCTURAL ANALYSIS}

\section{XRD Analysis}

Room temperature powder XRD patterns with their corresponding Rietveld refinement for $\mathrm{Mn}_{2-x} \mathrm{PtIn}$ with $\mathrm{x}=0.0$ to 1.0 are presented in Fig. 7 and Fig. 8. We have performed Rietveld refinement with the space group (SG) $\mathrm{I} \overline{\mathrm{m}} \mathrm{m} 2$ (SG No. 119) for $\mathrm{x}=0.0$ to 0.3 and $\mathrm{I} \overline{4} 2 \mathrm{~m}$ (SG No. 121) for $\mathrm{x}=0.5$ to 1.0. The parent compound $\mathrm{Mn}_{2} \mathrm{PtIn}$ crystallizes in the inverse Heusler tetragonal structure which belongs to the space group I $\overline{4} \mathrm{~m} 2$. In this structure the Mn atoms sits at the Wyckoff positions $2 \mathrm{~b}(0,0$, $0.5)$, and $2 \mathrm{~d}(0,0.5,0.75)$, whereas, In and Pt atoms occupies the $2 \mathrm{a}(0,0,0)$ and $2 \mathrm{c}(0,0.5,0.25)$, respectively. Samples with composition $\mathrm{x}=0.2$ to 0.4 show a structurally mixed-phase due to the composition-dependent structural transition from SG I $\overline{4} \mathrm{~m} 2$ to $\mathrm{I} \overline{4} 2 \mathrm{~m}$. Samples 


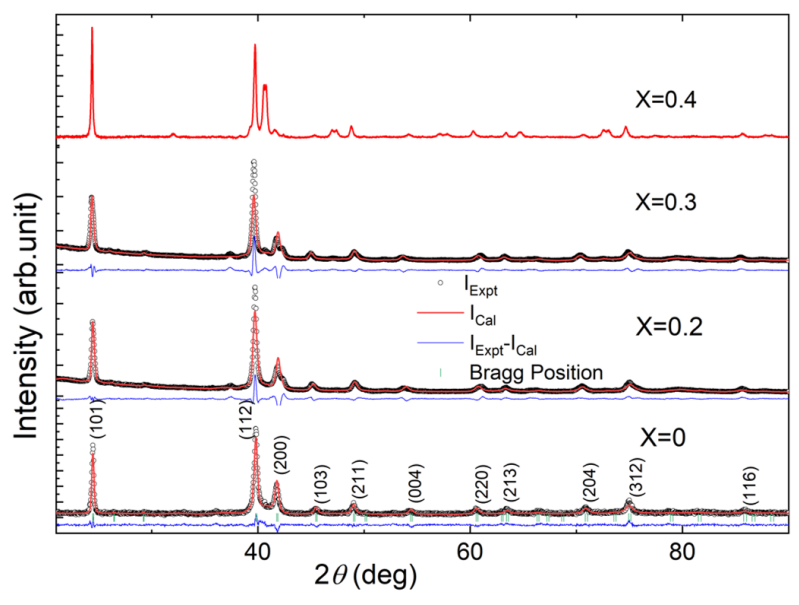

FIG. 7: (Color online) Room temperature XRD patterns for $\mathrm{Mn}_{2-x}$ PtIn for $\mathrm{x}=0.0$ to 0.4 . The open black circles represent the experimental data, the red color fitted lines to the experimental data represent the simulation pattern obtained from the Rietveld refinement. The difference between the experimental and simulation patterns is shown below the respective XRD patterns. The scattered vertical lines indicates Bragg's positions. The ( $\mathrm{h} \mathrm{k} \mathrm{l}$ ) value for all major reflections are shown for $\mathrm{x}=0.0$.

with the composition ranges $\mathrm{x}=0.5$ to 1.0 , also crystallize in tetragonal structure and belong to the space group I $\overline{4} 2 \mathrm{~m}$ (SG No. 121). Within this structure the Mn atoms occupy four different $\mathrm{Wyckoff}$ positions, i.e. $\mathrm{Mn}_{I}$ at $2 \mathrm{a}$ $(0,0,0), \mathrm{Mn}_{I I}$ at $2 \mathrm{~b}(0,0,0.5), \mathrm{Mn}_{I I I}$ at $4 \mathrm{~d}(0,0.5,0.25)$ and $\mathrm{Mn}_{I V}$ at $8 \mathrm{i}(\mathrm{x}, \mathrm{x}, \mathrm{z})$. Pt atoms occupies two Wyckoff sites $4 \mathrm{c}(0,0.5,0)$ and $4 \mathrm{e}(0,0,0.26473)$, whereas, In sits at the $8 \mathrm{i}\left(\mathrm{x}^{\prime}, \mathrm{x}^{\prime}, \mathrm{z}^{\prime}\right)$. Here it can be mentioned that a small fraction of $\mathrm{MnPt}$ phase (CuAu- I type tetragonal structure) 40 is observed for $\mathrm{x}=0.9$ and 1.0 samples. The MnPt phase fractions were estimated to be approximately $13 \%$ and $16 \%$ in the case of $\mathrm{Mn}_{1.1} \mathrm{PtIn}$ and $\mathrm{MnPtIn}$ respectively. Since $\mathrm{MnPt}$ shows an antiferromagnetic [40] ordering with zero net magnetic moments, it should not affect the magnetic properties of $\mathrm{Mn}_{1.1} \mathrm{PtIn}$ and MnPtIn samples. The structural and magnetic parameters of $\mathrm{Mn}_{2-x} \mathrm{PtIn}$ are summarized in Table-II.

\section{SEM and EDS Analysis}

The compositional homogeneity in the present samples were determined with help of Scanning Electron Microscopy (SEM). The SEM images for three samples that are mostly used for topological Hall effect measurement are shown in Fig. 9. The single-phase nature of the samples can be clearly seen from the SEM images. Small black spots in the Fig. 9(a) is due to the presence of small holes on the sample surface. To know the chemical composition of these samples we have performed energy dispersive X-ray analysis (EDS) at several places on

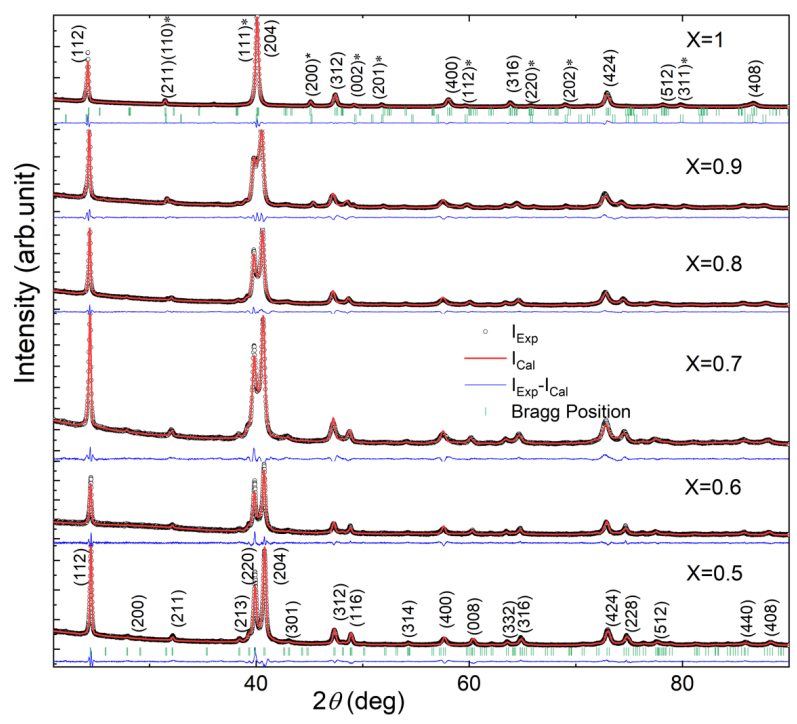

FIG. 8: (Color online) Room temperature XRD patterns for $\mathrm{Mn}_{2-x}$ PtIn for $\mathrm{x}=0.5$ to 1.0 . The open black circles represent the experimental data and the red color fitted lines to the experimental data after Rietveld refinement. The difference between the experimental and simulation patterns is shown below the respective XRD patterns. The scattered vertical lines indicates Bragg's positions. The ( $\mathrm{h} \mathrm{k} \mathrm{l}$ ) value for all major reflections are shown for $\mathrm{x}=0.5$ and 1.0. The $(\star)$ marked ( $\mathrm{h} \mathrm{k} \mathrm{l}$ ) indexes for $\mathrm{x}=1.0$ belongs to the $\mathrm{MnPt}$ secondary phase.

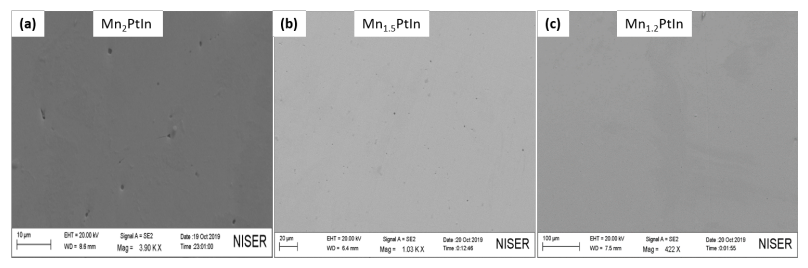

FIG. 9: (Color online) SEM images of (a) $\mathrm{Mn}_{2} \mathrm{PtIn}$, (b) $\mathrm{Mn}_{1.5}$ PtIn and (c) $\mathrm{Mn}_{1.2}$ PtIn.

the sample. As it can be seen from Table- I, the chemical composition obtained from the EDS analysis nearly matches with the starting composition of the samples.

\section{MAGNETIC STUDIES}

The temperature dependence of the magnetization for the $\mathrm{Mn}_{2-x} \mathrm{PtIn}$ for $\mathrm{x}=.0$ and $\mathrm{x}=0.5$ to 1.0 are shown in the main manuscript. As it can be seen, the Curie temperature systematically decreases with decreasing $\mathrm{Mn}$ concentration. We have summarized various parameters related to the structural and magnetic properties of the present samples in Table-II. Fig. 10(a)-(c) represents the first derivative of the $\mathrm{M}-\mathrm{H}$ data taken at $5 \mathrm{~K}$ for $\mathrm{Mn}_{2} \mathrm{PtIn}$, $\mathrm{Mn}_{1.5}$ PtIn and $\mathrm{Mn}_{1.2} \mathrm{PtIn}$ respectively. The DC mag- 
TABLE I: Comparison of exact stoichiometric atomic percentages with obtain data from EDS

\begin{tabular}{lll}
\hline Selected Composition & Exact atomic ratio (\%) & Obtained from EDS (\%) \\
\hline $\mathrm{Mn}_{2}$ PtIn & Mn-50, Pt-25, In-25 & Mn-52.03, Pt-25.62, In-22.34 \\
$\mathrm{Mn}_{1.5}$ PtIn & Mn-42.85, Pt- 28.57, In-28.57 & Mn-42.53, Pt-29.38, In-28.06 \\
$\mathrm{Mn}_{1.2}$ PtIn & Mn-37.50, Pt-31.25, In-31.25 & Mn-40.74, Pt-30.45, In-28.80 \\
\hline
\end{tabular}

TABLE II: Structural and magnetic parameters

\begin{tabular}{|c|c|c|c|c|c|c|}
\hline Composition(x) & $\begin{array}{l}\text { structure (Space } \\
\text { Group) }\end{array}$ & $a=b($ in $\AA)$ & $c($ in $\stackrel{\circ}{A})$ & c/a ratio & $T_{C}(\mathrm{~K})$ & $\begin{array}{l}M_{S} \quad\left(\mu_{B} / \text { f.u. }\right) \\
\text { Experimental } \\
\text { (at } 5 \text { T field) }\end{array}$ \\
\hline $\mathrm{x}=0.0$ & Tet. (İ̄̄m2) & $4.32(1)$ & $6.74(3)$ & 1.56 & 344 & 1.69 \\
\hline $\mathrm{x}=0.2$ & 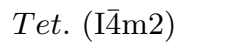 & $4.30(8)$ & $6.79(0)$ & 1.57 & 305 & 2.09 \\
\hline $\mathrm{x}=0.3$ & Tet. $(\mathrm{I} \overline{4} \mathrm{~m} 2)^{*}$ & $4.30(7)$ & $6.82(4)$ & 1.58 & 314 & 2.13 \\
\hline $\mathrm{x}=0.4$ & Tet. $(\mathrm{I} \overline{\mathrm{m}} \mathrm{m} 2)^{*}$ & $4.42(8)$ & $6.32(4)$ & 1.43 & 325 & 1.81 \\
\hline $\mathrm{x}=0.5$ & Tet. (İ̄ $2 \mathrm{~m})$ & $6.39(6)$ & $12.27(0)$ & 1.91 & 239 & 1.66 \\
\hline $\mathrm{x}=0.6$ & Tet. $(\mathrm{I} \overline{4} 2 \mathrm{~m})$ & $6.40(1)$ & $12.28(2)$ & 1.92 & 231 & 1.47 \\
\hline $\mathrm{x}=0.7$ & Tet. $(\mathrm{I} \overline{4} 2 \mathrm{~m})$ & $6.40(6)$ & $12.30(7)$ & 1.92 & 208 & 1.03 \\
\hline $\mathrm{x}=0.8$ & Tet. $(\mathrm{I} \overline{4} 2 \mathrm{~m})$ & $6.40(8)$ & $12.33(0)$ & 1.92 & 172 & 0.84 \\
\hline $\mathrm{x}=0.9$ & Tet. $(\mathrm{I} \overline{4} 2 \mathrm{~m})^{\dagger}$ & $6.40(3)$ & $12.37(1)$ & 1.93 & 141 & 0.44 \\
\hline $\mathrm{x}=1.0$ & Tet. $(\mathrm{I} \overline{4} 2 \mathrm{~m})^{\dagger}$ & $6.36(0)$ & $12.67(2)$ & 1.99 & 82 & 0.13 \\
\hline
\end{tabular}

netic field dependent AC susceptibility data of these samples also show similar behavior. Fig. 10(d) shows the second derivative of the magnetization with respect to the magnetic field. This gives the field at which the magnetization loop changes its slope just before going to a single domain state. The purpose is to compare the field obtained from the second derivative of magnetization with the field where we observe a maximum topological Hall effect.

\section{POWDER NEUTRON DIFFRACTION}

To determine the magnetic structure of the sample $\mathrm{Mn}_{1.5}$ PtInn we performed the powder neutron diffraction (ND) at different temperatures. The ND patterns taken in the temperature range of $1.5 \mathrm{~K}$ to $300 \mathrm{~K}$ are depicted in the main manuscript. The intensity variation of the different Braggs peaks with temperature is shown in the main text. The temperature dependent ND pattern shows increase in the scattering intensity below the ordering temperature at the nuclear Bragg peaks (101), (200) and (004), suggesting a commensurate magnetic structure. Furthermore, we obtained the magnetic propagation vector $\mathrm{k}=(0,0,0)$ with best agreement factors by using the k-search programme included in Fullprof-suite package. We used the previously determined space group I $\overline{4} 2 \mathrm{~m}$ (SG No. 121) and structural parameters for the Rietveld refinement. We have performed the Rietveld refinement of the $300 \mathrm{~K} \mathrm{ND}$ pattern using only the nuclear contribution. The fit parameters obtained from the $300 \mathrm{~K}$ refinement were used for the refinement of structural part for the low temperature data. The Rietveld refinement of 1.5 K ND data convincingly demonstrates the presence of magnetic contribution in $\mathrm{Mn}_{1.5} \mathrm{PtIn}$. The four magnetic atoms situated in the four different Wyckoff positions. It is difficult to have unique magnetic representations as the number of magnetic atoms is large. Therefore, we tried with different models obtained from symmetry analysis. The symmetry analysis of the magnetic structure was conducted by the software SARAh[41. Mn2a and Mn2b contained one 1-dim irreducible representations (IRs) Г2 one time and one $2 \mathrm{dim}$ irreducible representations (IRs) $\Gamma 5$ one time. Mn4d site with two 1-dim irreducible representations (IRs) $\Gamma 1$ and $\Gamma 2$ contained one time and one 2-dim irreducible representations (IRs) $\Gamma 5$ contains two times. Mn8i site with two 1-dim irreducible representations (IRs) $\Gamma 1$ and $\Gamma 3$ contained one time and two 1-dim irreducible representations (IRs) $\Gamma 2$ and $\Gamma 4$ contained 2 times. Also one 2 -dim irreducible representations (IRs) $\Gamma 5$ contains 3 times. The model that best describe the magnetic structure are in agreement with the irreducible representations (IRs) $\Gamma 5$ (Mn2a, Mn2b and Mn4d) while $\mathrm{Mn} 8 \mathrm{i}$ is represented by the superposition of irreducible representations (IRs) $\Gamma 5$ and $\Gamma 2$. The moment direction of Mn2a, Mn2b and Mn4d within the a-b plane are not fixed, therefore we fixed them parallel to the crystallohraphic [010] direction. Restricting the MX (Mn8i) = 0 , results in the non-collinear ferrimagnetic (we refer as model-1) ordering while the alternating $\mathrm{MX}(\mathrm{Mn} 8 \mathrm{i})$ component $(+\mathrm{x},-\mathrm{x})$ result into a non-coplanar structure (we refer as model-2)[Fig. 11]. In model- 1, Mn8i moment 

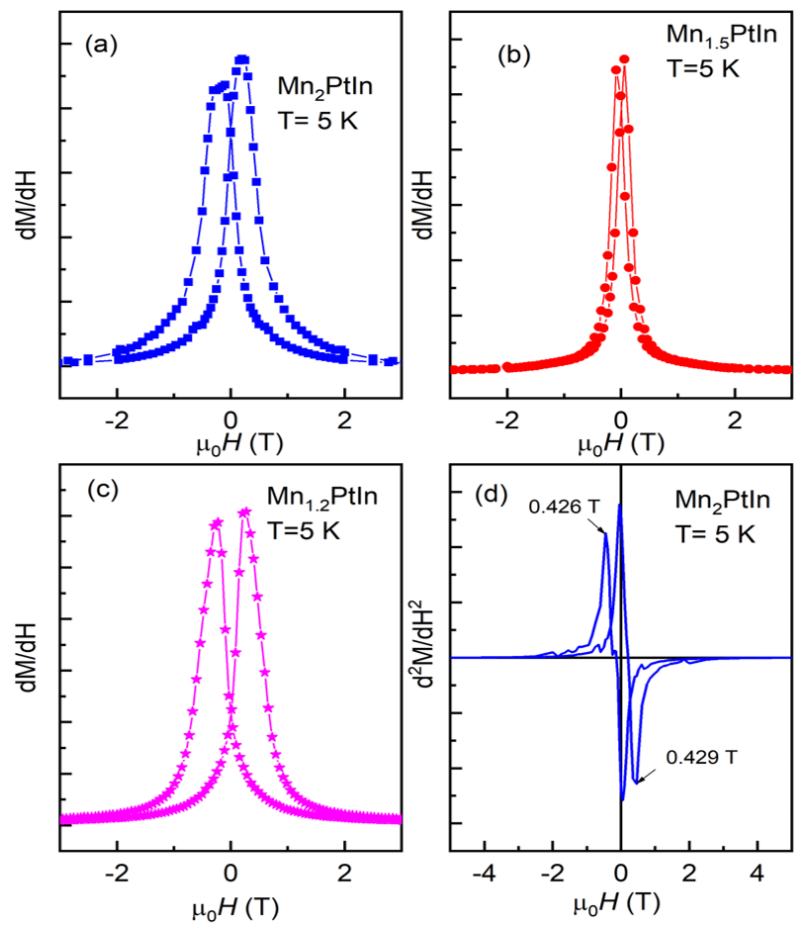

FIG. 10: (Color online) . The first derivative of the M-H data taken at $5 \mathrm{~K}$ for (a) $\mathrm{Mn}_{2} \mathrm{PtIn}$, (b) $\mathrm{Mn}_{1.5}$ PtIn and (c) $\mathrm{Mn}_{1.2}$ PtIn. (d) The second derivative of the M-H data taken at $5 \mathrm{~K}$ for $\mathrm{Mn}_{2} \mathrm{PtIn}$.

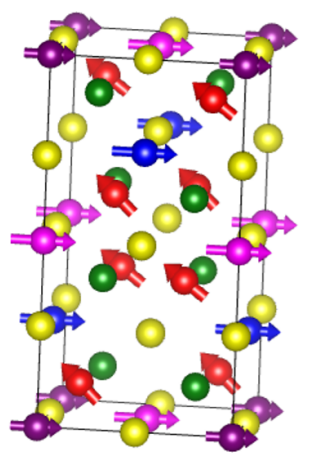

Model-1. Non-collinear

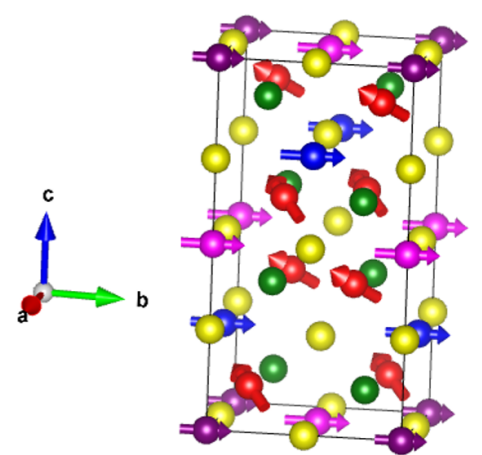

Model-2. Non-coplanar
FIG. 11: (Color online) Magnetic structure for two different model of $\mathrm{Mn}_{1.5} \mathrm{PtIn}$ at $1.5 \mathrm{~K}$. For the visual clarity the magnitude and direction of $\mathrm{Mn}(8 \mathrm{i})$ moments are not at per scale.

posses with the $\mathrm{M}_{Y}$ and $\mathrm{M}_{Z}$ component and for model-2 (non-oplanar structure) along with these two component additional $\mathrm{M}_{X}(\mathrm{Mn} 8 \mathrm{i})$ took alternate value (sequencing as $+\mathrm{x},-\mathrm{x})$. The refinement, by considering these two model shows very similar agreement as can be seen from the Fig. 12. The evidence of the non-collinear magnetic ordering is indiated by the increases in the intensity of the (101) and (004) Bragg peaks. The enhancement in

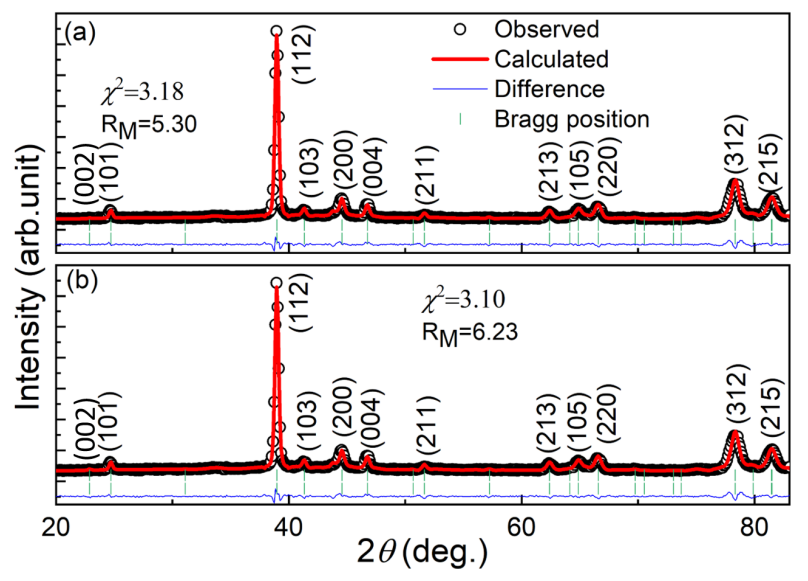

FIG. 12: (Color online) Rietveld refinement of the neutron diffraction patterns at $1.5 \mathrm{~K}$ for $\mathrm{Mn}_{1.5} \mathrm{PtIn}$ for two different model (a) non-collinear and (b) non-coplanar.

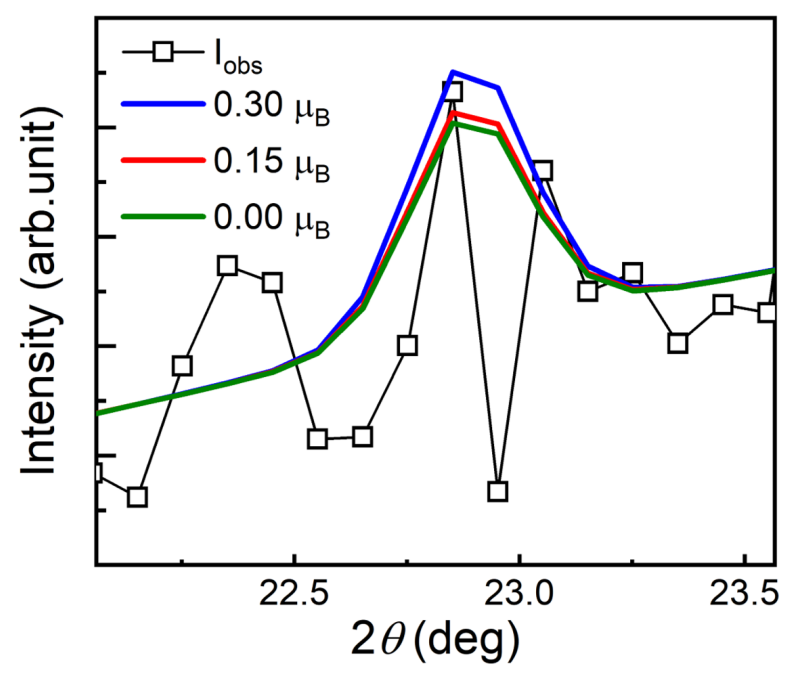

FIG. 13: (Color online) Evolution of the calculated intensity on top of the (002) Bragg peak for the different magnitude of $\mathrm{M}_{X}$ component of Mn8i moment.

the intensity of these Bragg peaks is forbidden if all the magnetic moment contained only in the crystallpgraphic [001] direction. However, the increment in the intensity of the (004) peaks also hints a non-coplanar ordering in this system. Again, the emergence of the (002) peak would have constitute the non-coplanar structure (reffered as model- 2) as a more favourable. This fact an be understood from the evolution of the calculated intensity of the (002) peaks with the variation of the magnitude of the $\mathrm{M}_{X}(\mathrm{Mn} 8 \mathrm{i})$ component as shown in Fig. 13. However, as shown in Fig. 13., the non-coplanar component might yield a very weak reflection which may not be separated from the background signal. Furthermore, the parameters that were varied during the refinement are the scale, 


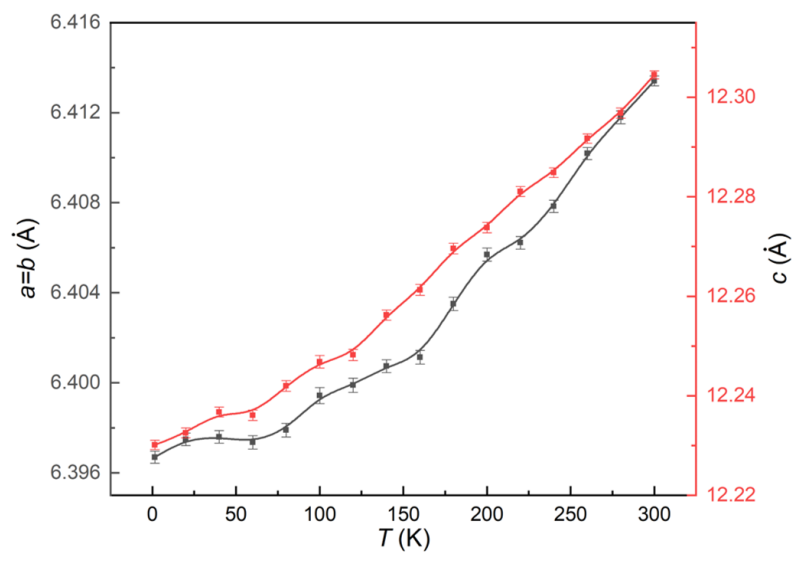

FIG. 14: (Color online) Variation of lattice parameters with temperature of $\mathrm{Mn}_{1.5} \mathrm{PtIn}$. The solid lines are guide to eye.

zero-shift, the lattice constants (a, c), projections of the spin $\left[\mathrm{M}_{Y}(\mathrm{Mn} 2 \mathrm{a}, \mathrm{Mn} 2 \mathrm{~b}, \mathrm{Mn} 4 \mathrm{~d}), \mathrm{M}_{Y}(\mathrm{Mn} 8 \mathrm{i}), \mathrm{M}_{X}(\mathrm{Mn} 8 \mathrm{i})\right.$, and $\mathrm{M}_{Z}(\mathrm{Mn} 8 \mathrm{i})$ ], and overall isotropic displacement (temperature) factor. Initially, we varied the parameter one by one and then releases some or all together at a time. The temperature dependence of absolute values of the magnetic moments for Mn sitting at different sublattices of $\mathrm{Mn}_{1.5} \mathrm{PtIn}$ are shown in the main mausript. The $\mathrm{Mn}$ sitting at $2 \mathrm{~b}, 4 \mathrm{~d}$ and $8 \mathrm{i}$ display almost equal magnitudes of magnetic moments with similar temperature dependance. A smaller magnitude of $2 \mathrm{a} \mathrm{Mn}$ moment is due to the fact that 2a site is comparatively less occupied in the present sample. For non-coplanar magnetic structure, the $\mathrm{Mn}(8 \mathrm{i})$ moment takes a alternate $\mathrm{M}_{X}$ component and we obtained small $\mathrm{M}_{X}(\mathrm{Mn} 8 \mathrm{i})=0.30(1) \mu_{B}$. The variation of the lattice constants with the temperature obtained from the neutron diffraction measurements is shown in the Fig. 14.

\section{HALL EFFECT MEASUREMENT}

The Hall Effect experiments were performed with rectangular shape Hall bars. Five probe Hall measurements were carried on the Hall bar samples of $\mathrm{Mn}_{2} \mathrm{PtIn}$, $\mathrm{Mn}_{1.5} \mathrm{PtIn}$ and $\mathrm{Mn}_{1.2} \mathrm{PtIn}$. The total Hall resistivity generally consists of two terms in trivial ferromagnetic/ferrimagnetic samples and can be expressed as $\rho_{y x}=\rho_{N}+$ $\rho_{A H}$, where $\rho_{N}$ and $\rho_{A H}$ are the normal and anomalous Hall $(\mathrm{AH})$ resistivity respectively. Normal Hall $\left(\rho_{N}\right)$ is given by $\rho_{N}=R_{0} \mathrm{H}$, where $R_{0}$ is the normal Hall coefficient and related to the carriers density of the materials and $\mathrm{H}$ is the external magnetic field. However, the origin of anomalous Hall $\left(\rho_{A H}\right)$ effect evolves with time since the discovery of it and can be classified into two mechanisms (i) intrinsic and (ii) extrinsic. The notion of Berryphase curvatures comes under the intrinsic mechanism,

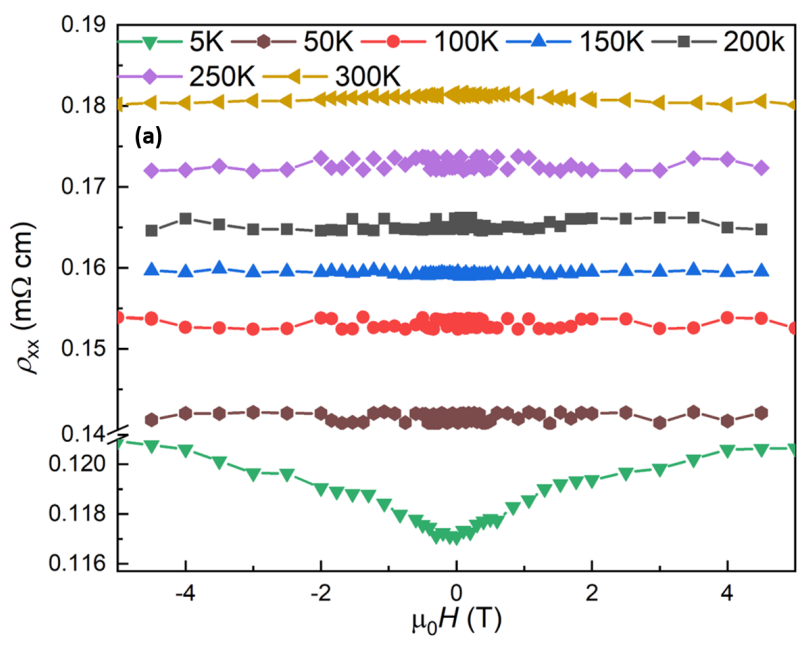

FIG. 15: (Color online) Field dependence of the longitudinal resistivity $\left(\rho_{x x}\right)$ for $\mathrm{Mn}_{2} \mathrm{PtIn}$ at various temperatures.
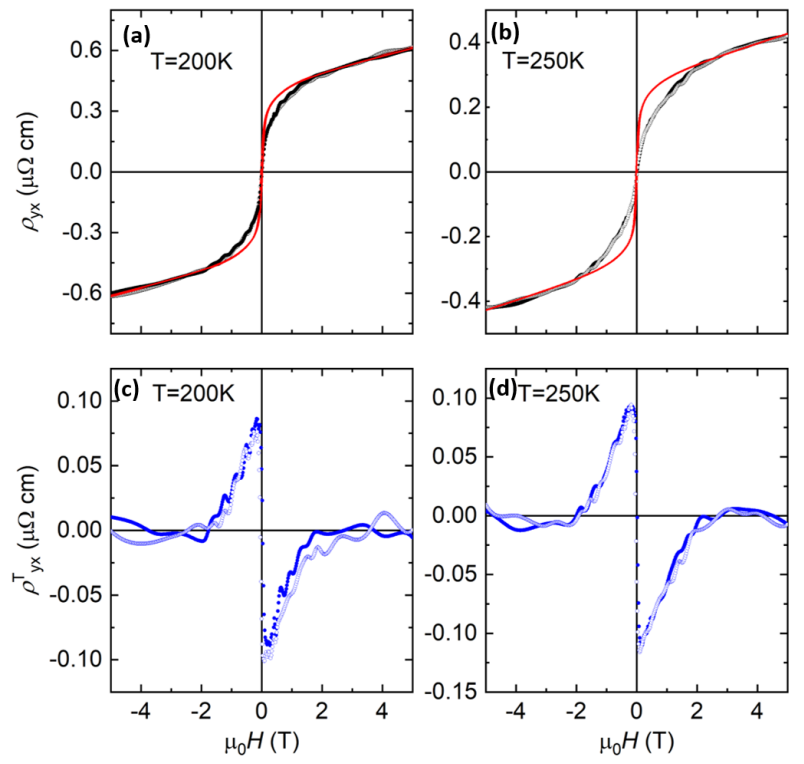

FIG. 16: (Color online) Experimental and calculated Hall resistivity of $\mathrm{Mn}_{2} \mathrm{PtIn}$. (a) and (b) experimental (black open $(+5 \mathrm{~T}$ to $-5 \mathrm{~T})$ and solid $(-5 \mathrm{~T}$ to $+5 \mathrm{~T})$ symbols) and calculated (solid red line) Hall resistivity curves at temperatures $200 \mathrm{~K}$ and $250 \mathrm{~K}$ respectively of the sample $\mathrm{Mn}_{2} \mathrm{PtIn}$. (c) and (d) corresponding to the topological Hall resistivity at different temperatures. Open symbols for $+5 \mathrm{~T}$ to $-5 \mathrm{~T}$ and solid symbols for $-5 \mathrm{~T}$ to $+5 \mathrm{~T}$.

whereas, the skew scattering and side-jump belongs to the extrinsic mechanism 42 .

It is noteworthy to mention that the AH resistivity arise from the intrinsic mechanism varies as square of the longitudinal resistivity $\left(\rho_{x x}\right)$ and directly scales with the magnetization $(\mathrm{M})$ of the sample and can be written as $\rho_{A H}=\mathrm{b} \rho_{x x}^{2} \mathrm{M}$, where $\mathrm{b}$ is the constant. However, the ex- 


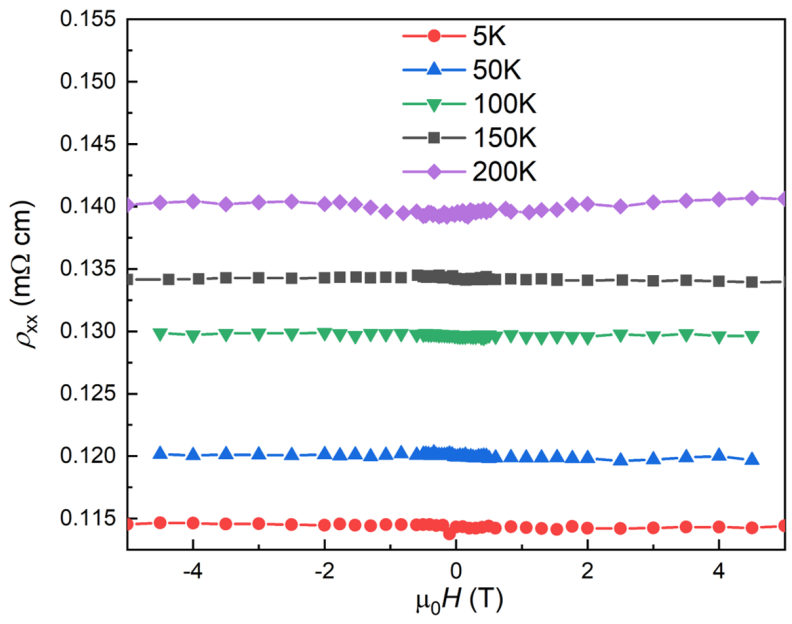

FIG. 17: (Color online) Magnetic field dependence of the longitudinal resistivity $\left(\rho_{x x}\right)$ for $\mathrm{Mn}_{1.5} \mathrm{PtIn}$ at various temperatures.
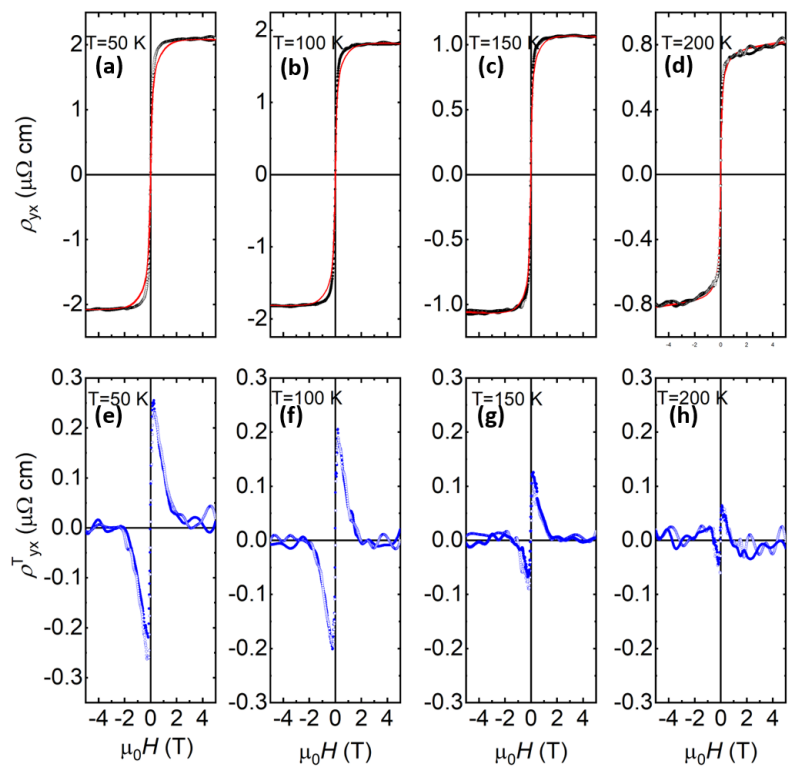

FIG. 18: (Color online) Experimental and calculated Hall resistivity of $\mathrm{Mn}_{1.5} \mathrm{PtIn}$. (a), (b), (c) and (d) Experimental (black open $(+5 \mathrm{~T}$ to $-5 \mathrm{~T})$ and solid $(-5 \mathrm{~T}$ to $+5 \mathrm{~T})$ symbols) and calculated (solid red line) Hall resistivity curves at different temperatures for the sample $\mathrm{Mn}_{1.5} \mathrm{PtIn}$. (e), (f), (g), and $(\mathrm{h})$ corresponding to the topological Hall resistivity at different temperatures.

trinsic contribution from the skew scattering $\left(\rho_{A H}^{\text {skew }}\right)$ and side jump $\left(\rho_{A H}^{s j}\right)$ to the $\mathrm{AH}$ resistivity can be express in terms of the longitudinal resistivity $\left(\rho_{x x}\right)$ such as $\rho_{A H}^{\text {skew }}$ $\propto \rho_{x x}$ and $\rho_{A H}^{s j} \propto \rho_{x x}^{2}$ 42. Also, a new scaling relation demonstarted for $\rho_{A H}^{s k e w}$ and $\rho_{A H}^{s j}$ for high conductivity materials $\left(\sigma_{x x} \sim 10^{6} \Omega^{-1} \mathrm{~cm}^{-1}\right)$ in terms of the residual resistivity $\left(\rho_{x x 0}\right)$ instead of $\rho_{x x}$ [4]. Furthermore,

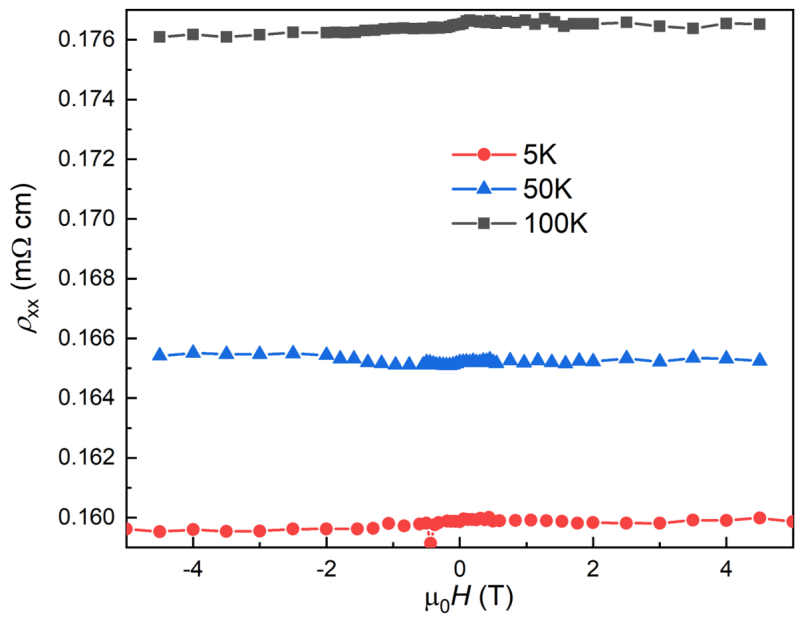

FIG. 19: (Color online) Field dependence of the longitudinal resistivity $\left(\rho_{x x}\right)$ for $\mathrm{Mn}_{1.2}$ PtIn at various temperatures.
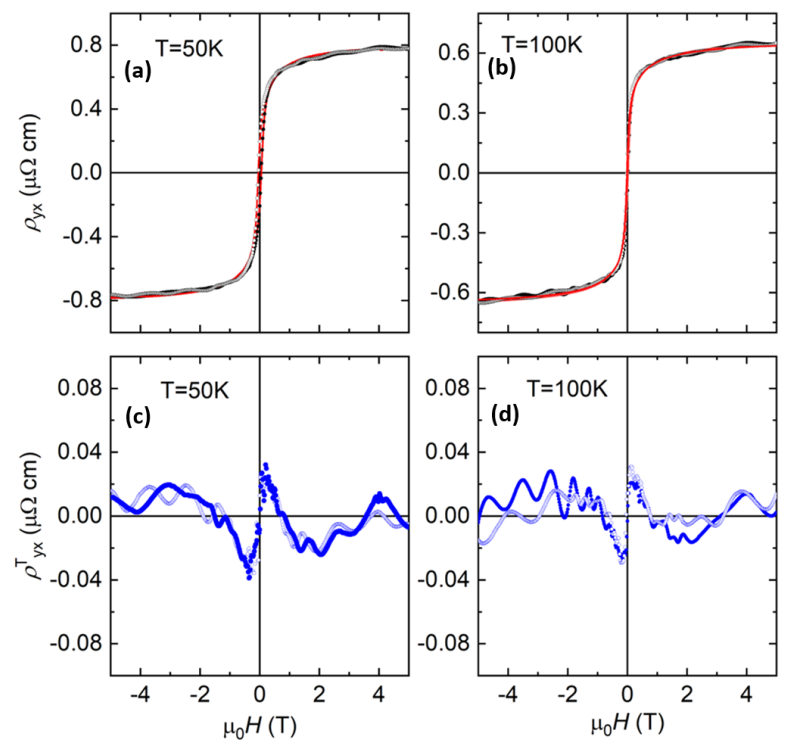

FIG. 20: (Color online) Experimental and calculated Hall resistivity of $\mathrm{Mn}_{1.2} \mathrm{PtIn}$. (a) and (b) experimental (black open $(+5 \mathrm{~T}$ to $-5 \mathrm{~T})$ and solid $(-5 \mathrm{~T}$ to $+5 \mathrm{~T})$ symbols $)$ and calculated (solid red line) Hall resistivity curves at different temperatures $50 \mathrm{~K}$ and $100 \mathrm{~K}$ respectively of the sample $\mathrm{Mn}_{1.2} \mathrm{PtIn}$. (c) and (d) corresponding to the topological Hall resistivity at different temperatures. Open and solid circles corresponding to $+5 \mathrm{~T}$ to $-5 \mathrm{~T}$ and $-5 \mathrm{~T}$ to $+5 \mathrm{~T}$ respectively.

different regime have been suggested to distinguish the dominant contribution from intrinsic or skew scattering process to AH resistivity based on the conductivity of the system under study [42. The anomalous Hall effect due to skew scattering $\left(\rho_{A H}^{\text {skew }}\right)$ and side jump $\left(\rho_{A H}^{s j}\right)$ terms will be irrelevant even at low temperatures in materials with moderate conductivity $\left(10^{4}\right.$ to $\left.10^{6} \Omega^{-1} \mathrm{~cm}^{-1}\right)$ with negligible magnetoresistance [15, 44,46. All the materi- 
als studied here falls under the moderate conductivity regime. So, $\rho_{y x}$ can be written as $\rho_{y x}=R_{0} \mathrm{H}+\mathrm{b} \rho_{x x}^{2} \mathrm{M}$ at the high field. The unknown constant $R_{0}$ and $\mathrm{b}$ is obtained by a straight line $(y=\mathrm{m} x+\mathrm{c})$ fitting in the plot of $\left[\rho_{y x} / \mathrm{H}\right.$ versus $\left.\left(\rho_{x x}^{2} \mathrm{M}\right) / \mathrm{H}\right]$. The obtained parameters are utilized to calculate the complete Hall loop. As it can be seen from the Fig. 3 of the main manuscript that the calculated Hall resistivity matches perfectly with the experimental data at the high fields by considering only the intrinsic contribution to the anomalous Hall. The calculated Hall resistivity subtracted from the experimental
Hall data to obtained the topological Hall contribution. Here it can be mentioned that taking only the intrinsic contribution in the anomalous Hall, the calculated and the experimental Hall resistivity data coinside with each other for $\mathrm{Mn}_{1.2} \mathrm{PtIn}$ in all field regions. However, an adequate difference sustained for $\mathrm{Mn}_{2} \mathrm{PtIn}$ and $\mathrm{Mn}_{1.5} \mathrm{PtIn}$ in the low field regime. Figs. 15-20. show the field dependence of longitudinal resistivity and Hall resistivity for $\mathrm{Mn}_{2} \mathrm{PtIn}, \mathrm{Mn}_{1.5} \mathrm{PtIn}$ and $\mathrm{Mn}_{1.2} \mathrm{PtIn}$ at various temperatures. 